\title{
Téoros
}

Revue de recherche en tourisme

\section{Vers un nouveau mode de détermination des conditions de} travail

\section{Michel Grant}

Volume 2, numéro 1, février 1983

Formation et conditions de travail en tourisme

URI : https://id.erudit.org/iderudit/1080839ar

DOI : https://doi.org/10.7202/1080839ar

Aller au sommaire du numéro

Éditeur(s)

Université du Québec à Montréal

ISSN

0712-8657 (imprimé)

1923-2705 (numérique)

Découvrir la revue

Citer cet article

Grant, M. (1983). Vers un nouveau mode de détermination des conditions de travail. Téoros, 2(1), 14-19. https://doi.org/10.7202/1080839ar d'utilisation que vous pouvez consulter en ligne.

https://apropos.erudit.org/fr/usagers/politique-dutilisation/ 


\title{
Vers un nouveau mode de détermination des conditions de travail
}

\author{
par Michel Grant*
}

Lors d'une conférence sectorielle sur le toutisme tenue en 1978, le document de réflexion déposé par le gouvernement du Québec décrivait les conditions de travail du personnel oeuvrant dans l'industrie touristique comme étant inférieures à celles prévalant dans la grande majorité des secteurs d'activités économiques ${ }^{i !}$. La plupart d'entre nous, subissons directement ou indirectement les effets de la mauvaise conjoncture économique actuelle. Plusieurs groupes de salariésles), même syndiqués(es), voient leur pouvoir d'achat diminuer et parfois même leur salaire subir des coupures. La situation est donc difficile pour tout le monde, mais elle l'est d'autant plus pour ceux et celles qui travaillent dans l'industrie du tourisme; en effet, ces derniers se trouvent dans une position qui, dejà avant la crise que nous traversons, les défavorisait sur le plan des conditions de travail et que la situation economique actuelle ne fait qu'aggraver.

Très peu de recherches ont été réalisées sur le problème des conditions de travail et de la syndicalisation dans lindustrie touristique et cet état de fait n'a pas été sans créer de difficultés dans la préparation du présent article. Nous allons quand mème tenter de dresser un tableau tres sommaire de la situa tion de l'emploi et des conditions de travail dans ce secteur et de dégager des avenues possibles quant à un mode éventuel de determination des dites conditions.

Nous esperions que ce bref survol de la question incitera d'autres personnes à pousser plus loin la recherche et l'analyse. Nous avons en effet constate des lacunes impoi. tantes au niveau de l'accessibilite et de lexistence même de données statistiques précises et détaillees sur l'emploi et les conditions de travail dans l'industrie du tourisme. Les sources d'informations auxquel-

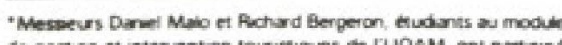
de pesion et intervention tourificues de rugaM, ont purticipt

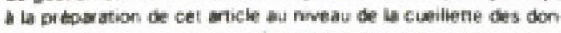

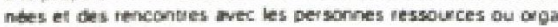
nomes wotcialut les nous avons pu avoir accès et les chiffres que nous avons pu obtenir demeurent épars et incomplets. A part les sources habituelles lex:- Statistique Canada et lies publications specialisees de divers groupesi, nous avons rencontré ou contacté les organismes énumérés plus bas ${ }^{(0)}$ afin de dresser le tableau le plus conforme possible à la réalité.

\section{L'industrie touristique: un secteur non-intégré}

Comme le souligne monsieur Claude Jouhannet dans son article du présent numéro de Téoros, l'industrie du tourisme comprend plusieurs activités qui sont reliées à d'autres secteurs, comme ceux de l'hôtellerie et de la restauration, du commerce de detail et du transport. C'est sans doute là une des raisons qui explique l'absence du tourisme dans la liste des secteurs productifs au Québec ${ }^{\mathrm{Ca}}$ et que celui-ci ne fait pas l'objet d'une comptabilité économique et statistique distincte. Ainsi, malgré toute la rigueur scientifique des travaux de recherche effectués jusqu'à maintenant, nous ne disposons que d'estimations sur le nombre d'emplois et les revenus engendrés par les dépenses touristiques. II faut toutefois reconnaitre quil est très difficile d'identifier et de comptabiliser des emplois consacrés exclusivement et d'une façon permanente au tourisme. Le rapport Baumann affirmait d'ailleurs en 1970 que le nombre d'emplois de ce type était bien minime; on y ajoutait même que:

"On pourrait mème en déduire qu'actuel. Jement en dehors des services gouverne. mentaux, des offices de tourisme municipaux et de certains services touristiques dans le transport aerien ou par autobus, ainsi que des sections de tourisme des gands quotidiens et des agences de voyage, il n'y a pratiquement pas d'orga: nismes ou d'entreprises qui puissent utiliser à lanné longue les services de personnes specialises uniquement en tourisme. c'est"ä-dire sans étre polyvalentes dans d'autres domaines techniques connexes ou non au tourisme".
Pour les fins de notre présentation, nous avons exclu les emplois reliés au tourisme qui se retrouvent dans les services publics. Leur nombre demeure relativement restreint et les conditions de travail sont pour la grande majorite definies par des conventions collectives. Quant au personnel des agences de voyage, les représentants de Statistique Canada nous ont informé que cette categorie ne fait pas l'objet elle non plus d'une classification specifique. Etant donné qu'il s'agit d'un secteur directement et exclusivement relie au tourisme et qu't semble exister des problemes au niveau des conditions de travail et de leur fixation ${ }^{15}$ ! nous avons essave de poursuivre plus loin notre "chasse" aux données. Selon deux sources d'informations ${ }^{16}$, nous pensons pouvoir affirmer que le nombre de person. nes qui y oeuvrent se situe à environ 4000 reparties dans 916 établissements au 31 décembre 1982 Igrossistes, détaillants et transporteurs). La proportion des femmes etait de $65 \%$ au 1er auril 1982 .

Compte-tenu des réserves mentionnees plus haut quant aux difficultés d'accès à des sta tistiques spécifiques et ventilèes, la recherche de Gosselin et Gagné nous amène à constater que la principale concentration d'emplois génères par les dépenses touristiques se situe dans le secteur de l'hotellerie et de la restauration. Ainsi, sur les 23,797 emplais directement supportés par ces dépenses en 1977, on en retrouvait 14,273 dans le secteur de l'hótellerie et de la restauration, soit $60 \% \%$. De plus, sur les 37,877 emplois générés directement ou indirectement par le tourisme, on en retrouvait 14,359 dans ce secteur en $1977^{18}$. Nous pouvons donc inclure que non seulement la part du secteur hôtellerie et restauration dans la création directe d'emplois est lá plus importante $(14,273$ $14,359=99 \%$ ), c'est-à-dire que la quasitotalité des emplois générés par les dépenses touristiques dans ce secteur sont relies directement aux activités touristiques, mais que de plus, c'est encore dans ce secteur qu'on retrouve le plus grand nombre d'emplois creés par ces depenses ${ }^{(2)}$. 
Les travailleurs et les travailieuses de lindustrie touristique exercent donc leur metier dans le cadre d'entreprises dont, pour la très grande majorité d'entre elles, la vocation n'est pas exclusivement ou méme principa lement consacree aux activités touristiques. Ces personnes font partie de groupes de salariésies) travailiant pour des employeurs bien distincts et dans des secteurs parfois differents. A des degres divers et selon des périodes variant avec les saisons, elles exé cutent des tâches en relation avec le tourisme. Pour les fins d'identification de leurs conditions de travail, on ne peut parler de ces personnes comme à l'emploi de l'industrie touristique. En effet, lorsqu'il s'agit de contrat de travail, que ce dernier soit individuel ou collectif, il existe de droit et de fait un emploveur qui a procéde à l'embauche d'un(e) salarié(e) dont il s'attend a une prestation de travail moyennant remunèration. Comme l'essentiel de nos préoccupations s'axe autour des relations du travail, nous percevons le secteur de l'hótellerie et de la restauration comme un ensemble d'emploveurs ayant à leur service des salariètes] et reconnaissent à ceux-ci certaines conditions de travail dans un cadre qui fera l'objet de notre présentation.

\section{Emplois, employés(es) et conditions de travail dans le secteur de l'hótellerie et de la restauration}

Nous ne prétendons pas ici dresser un tableau complet de la structure occupationnelle de l'emploi dans le secteur de l'hótel. lerie et de la restauration. De toute façon, nous ne disposons pas de données qui nous permettraient de réaliser une telle entreprise, particulièrement dans le cas de la restauration; comme nous allons le voir plus loin, des études un peu plus poussees ont été réalisées pour I'hótellerie. Quant à la nomenclature et á la typologie de l'emploi dans ce dernier secteur, nous rétèrons à l'article de $M$. Jouhannet Nous nous intéressons ici au nombre d'employés dans le secteur de l'hôtellerie et de la restauration au Quebec. à leurs principales caracteristiques et á leurs conditions de travail.

\subsection{Nombre d'employés et caractéristiques}

Selon Statistique Canada ${ }^{10}$, on retrouvait 53,300 salariés(es) dans !e secteur des hôttels, restaurants et tavernes en juin 1982. Cette donnée sous-estime largement la réa. lité et s'explique par la méthode d'enquéte utilisée par Statistique Canada qui exclut tous les employeurs ayant moins de 20 personnes a leur service ${ }^{\prime 11 !}$. Ces chiftres apparaissent d'autant plus conservateurs que le ministère du Revenu dénombrait à 210,976 les emplois dans le mème secteur en $1979^{112 !}$. Compte tenu du peu d'augmenta tion de l'emploi dans ce secteur, ces statistiques nous apparaissent irrealistes quand on les compare à d'autres sources d'information qui sont plus concordantes et dont la fiabilité nous semble plus conforme aux besoins de notre démarche, à savoir la déter- mination du nombre de personnes occupant des postes de niveau syndicable dans le sec. teur de l'hötellerie et de la restauration.

Ainsi l'emploi hôtelier au Québec regroupait en 197629.949 personnes $^{113}$ et en 1980 30,518 personnes ${ }^{114}$. On voit qu'il $y$ a peu de variation dans l'emploi et qu'on est loin des 48,643 emplois denombrés par le minis. tère du Revenu pour le secteur hébergement $t^{115}$. Selon une étude du Conseil du statut de la fermme basee sur les données obtenue des services de l'enquête sur la population active du gouvernement fede ral, on comptait au Québec 109,000 salariés(es) travaillant dans le secteur de l'hé bergement et de la restauration en $1976^{116}$. Se basant sur ces donnèes de 1976, nous pensons pouvoir ètre justifié de dèduire qu'on retrouvait alors 79,051 salariés(es) de la restauration, soit $72.5 \%$ de l'emploi dans l'ensemble de l'hótellerie et de la restaura tion; il s'agit donc d'un groupe important pour lequel nous ne possédons que très peu d'informations. Sous réserve des commen taires que nous émettions à l'égard de ces données, nous constatons que, dans les tableaux statistiques du ministère du Revenu, la proportion des emploi reliés à la restauration representait en $197977 \%$ de l'emploi dans le secteur hótellerie. restauration ${ }^{177}$, donc un pourcentage rela. tivement similaire à celui que nous déga gons plus haut.

Au niveau des recoupements de la main d'oeuvre par sexe, Statistique Canada indi. quait qu'en juin $1982,44 \%$ des salariésles) du secteur de l'hótellerie, restaurants et tavernes étaient des femmes ${ }^{178}$. Les données de 1976 sur lesquelles s'est basé le Conseil du Statut de la femme nous informent que les femmes occupaient $54 \%$ des emplais dans le secteur de l'hébergement et de la restauration ${ }^{179}$. Dans le secteur de I'hótellerie exclusivement, les femmes occupaient $51 \%$ des emplois $20 \%$; on souligne que la main-d'oeuvre féminine est plus importante proportionnellement dans les petits hŏtels que dans les grands hôtels et que

"Les emplois occupés majoritairement par des femmes sont, pour toutes les tailles d'hôtel, des occupations "d'entretien leger" $[211$

On peut également ajouter que les femmes se retrouvent surtout dans les emplois moins bien rémunérés par rapport à ceux occupés par les hommes.

Au niveau de la scolarité, $38 \%$ du personnel hótelier avait complété en 1978 un secondaire général $26 \%$ les études primai res et $17 \%$ un secondaire professionnel ${ }^{122}$

Quant à l'åge, on constatait en 1978, que $57.4 \%$ se situait dans la catégorie 25 ans .44 ans $^{123}$
Les autres particularites du secteur de lhò tellerie et de la restauration sont reliees au caractère saisonnier de l'emploi, au taux de roulement élevé du personnel, à la faible spécialisation professionnelle, à l'importance de la main-d'oeuvre immigrante ainsi qu'à une proportion importante de salariesiesl à temps partie $\left.\left.\right|^{\mid 241}\right)$. Ainsi selon des donnees non publiées du Bureau de la Statistique du Québec, les emplois à temps partiel dans le secteur de l'hótellerie représentaient en $198026.2 \%$ du total des emplois dans ce secteur alors qu'ils en representaient $21.4 \%$ en 1976 et que dutant cette méme dernière année, le pourcentage des emplois à temps partiel pour l'ensemble du Quebec n'était que de $8 \%$ (25).

Dans cette classification "temps plein ws temps partiel" basee sur celle du Bureau de la Statistique du Québec, on ne distingue pas les emplois de type occasionnel ou temporaire; la classification du B.S.Q implique que les emplois de type temporaire sont inclus dans la catégorie temps plein ou dans la catégorie temps partiel selon que le nombre d'heures travaillees durant la semaine est infêrieure ou non à 35 heures $5^{(26)}$. Ainsi en 1978, selon l'étude du Centre de recherche et de statistiques sur le marché du travail, les pourcentages de repartition de la main-d'oeuvre hótelière fluctuaient de la façon suivante à mesure que la taille des éta blissements s'élargissait: les emplois réguliers plein temps variaient de $62.8 \%$ à $87.6 \%$ de l'ensemble de la main-d'oeuvre hòtelière. les emplais reguliers temps partiel de $18.1 \%$ à $8.1 \%$ et les emplois temporaires de $19.1 \%$ à $4.3 \%(27)$

Les grands établissements ont donc plus tendance à maintenir un niveau d'emploi régulier à plein temps, sauf qu'en 1976 les établissements höteliers de moins de 49 chambres regroupaient $44 \%$ des emplois du secteur, et les etablissements de 99 cham. bres et moins $55.6 \%$. C'est donc là une réalité extrêmement importante dont doit tenir compte tout débat sur le mode de determination des conditions de travail. Même si nous n'avons pu ávoir accès à des données sur le secteur de la restauration comme telle, à l'exception évidemment de celui qu'on retrouve dans les hótels, nous savons que la distribution de la main-d'oeuvre y est encore plus déconcentrée que dans le secteut de l'hótellerie et que la problema tique de la qualité et du mode de determination des conditions de travail se pose avec encore plus d'ampleur que dans le secteur de l'hơtellerie.

\subsection{Les conditions de travail}

La très grande majorité des observateurs reconnaissent que les travailleurs et les travailleuses du secteur des hòteis et des res. taurants vivent des conditions de travail inferieures a celles prévalant sur le marchétal. Ainsi sur le plan salarial, en juin 1982, la rémuneration horaire moyenine était de $\$ 5,29$ dans le secteur des hötels, restaurants 
et tavernes alors qu'elle était de $\$ 9.48$ dans le secteur manufacturier à la même date ${ }^{(30)}$ Si on examine la répartition des taux horaires moyens par taille d'établissements, on constate une corrélation positive entre ces deux variables: plus la taille de l'établissement hôtelier est importante, plus le taux horaire moven est élevécil!. L'explication de ce phénomène n'est. pas uniquement attribuable à la taille de l'établissement comme tel mais aussi au fait que le syndicalisme a pénétré dans les établissements hóteliers de taille importante.

Quant au mode de rémunération, près du tiers du personnel hôtelier reçoit des pour boires. Dans le secteur de la restauration on ne retrouve pas des catégories de person. nel comme celles affectées à la réception et à l'intendance lex. entretien des cham. bresl dont une très forte proportion n'est pas soumise à une rémuneration au pourboire. Le pourcentage du personnel au pourboire est encore plus élevé dans le secteur de la restauration que dans celui de l'hottellerie (ex, serveuses). Dans ces cas la loi sur les normes du travail prevoit un taux horaire inférieure à celui stipulé pour l'ensemble des salariés(es) au Québec, soit $\$ 3.28$ au lieu de $\$ 4.00$. Nous ne voulons pas nous lancer ici dans toute la problematique soulevée par le Livre vert du ministère du Revenu sur les travailleursleusesl au pourboire, mais nous devons souligner, pour les fins du present article, que ces personnes sont particulierement pénalisées à cause de cette forme de rémunération au niveau des régimes publics de rente, d'accidents de travail et d'assu: rance automobile; en effet, les prestations prévues par ces régimes IR.R.Q., C.S.S.T. et R.A.A.Q.) sont basées en fait sur le salaire minimum ${ }^{(32)}$. Cette situation n'est pas cor. rigée par l'existence de régimes complémentaires d'avantages sociaux au niveau de l'établissement hôtelier ou du restaurant puisque

*... les programmes d'avantages sociaux offerts par les entreprises du secteur de I'hótellerie ef de la restauration sont tres restreints et reduits a leur plus simple expression" asi!

Parmi diautres conditions détavorables subies par ces salarieslesi, le Livie vert men tionne les congediements arbitraires, le partage des pourboires avec d'autres employés(es), le paiement de notes de repas egarées et le temps supplémentaire payé au taux horaire regulier ${ }^{134}$ ?

L'ensemble des salariès(es) du secteur de I'hótellerie et de la restauration est soumis a des horaires differents; ainsi $35 \%$ des employés(es) affectés(esl aux services hòteliers travaillent de 16 heures à 24 heures et $15 \%$ de 0 à 8 heures ${ }^{\mid 35}$. Certains emploveurs recourent â la formule des horaires brisés ou de rotation des horaires de travail. La nature mème du service demandé par la clientèle implique une présence impor- tante du personnel les samedi, dimanche et jours feries. Les possibilités de promotion sont minces, on constate en effet

"peu de mobilité occupationnelle a linté. rieur d'un méme établissement apres que Findividu ait ete embauche"r

II n'est donc pas surprenant que la direction des établissements hóteliers ait des proble. mes de rétention de sa main-d'oeuvre. Des qu'une personne peut se trouver un emploi qui offre de meilleurs avantages, elle quitte son emploi.

"Le développement de conditions de tra. vail concurrentielles simpose aussi pour diminuer les departs de la main-d'oeuvre... Les problèmes de recrutement dans le secteur hôtelier relevent davantage de la gestion des ressources humaines que de lab. sence d'une main-d"oeuvre qui desire travailler dans / hótellerie mais il faut que les etablissements höteliers soient capables de la retenir par des politiques de formation, de plan de carriere et de rémunération appropriées ${ }^{\text {nor }}$ gir.

\section{La détermination des conditions de travail}

L'encadrement prévu par la législation du travail au Quebec définit trois modes de détermination des conditions de travail. Le premier est celui où l'Etat decrète lui-mème les conditons de travail. Le second est celui oủ les parties, syndicale et patronale, définissent conjointement par négociation le contenu ds conditions de travail qui seront consignées dans une convention collective. Le troisiême mode est celui prévu en vertu de la Loi sur les decrets de convention collective. Dans ce dernier cas, le ministre du Travail peut decider d'extentionner en tout ou en partie une convention collective à tous les salariés(es) non syndiqués(es) dans un secteur donné d'activites. C'est un comité paritaire composé de representants du patronat et des syndiquéslesl qui voit à l'application des dispositions du décret. II existe une cinquantaine de decrets tels le decret de la confection du vêtement pour dames ou celui de la coiffure. Un decret s'applique donc dans un secteur d'activités bien déterminé et le ministre peut lui donner une application provinciale ou régionale. L'existence d'un décret implique donc au prealable une certaine présence syndicale dans le secteur concerné

\subsection{Loi sur les normes du travail}

C'est surtout dans cette loi qu'on retrouve la plupart des principales conditions minimales de travail et que l'Etat assure une certaine protection aux travailleurs et aux tra vailleuses de l'hótellerie et de la restauration. La Loi sur les normes du travail prevoit un salaire minimum qui est différent pour ceux qui travaillent au pourboire, des jours féries. des vacances annuelles, un droit de préa vis dans des cas de licenciement, une semaine de travail de 44 heures, etc, ainsi que certains recours dans les cas de congédiement. Le legislateur $n$ "a jamais pré tendu que celle loi allait constituer un subs. titut à la negociation collective. II s'agissait plutốt d'établir un certain plancher. D'autres légisiations interviennent à des degrés divers dans la determination des conditions de tra vail telles la Loi sur la Fête nationale ou la Loi sur la santé et la securité du travail.

Tout en assurant une protection minimale pour les salaries(es) de l'hôtellerie et de la restauration, ces lois ne constituent pas des instruments vraiment efficaces qui vont leu permettre d'améliorer d'une façon significa tive leurs conditions de travail et de se situer à un niveau comparatif plus avantageux que celui qu'ils connaissent actuellement. On ne peut donc s'attendre a une intervention de I'Etat pour relever le niveau des conditions minimales.

Diverses recherches nous indiquent que la syndicalisation est habituellement associee à un degré plus élevé de rémuneration et des avantages sociaux, et ce pour des emplois comparables ${ }^{3 \dot{s}}$. Ainsi l'étude du Conseil du statut de la femme établissait à $18 \%$ l'écart de la rémunération moyenne incluant les avantages sociaux en faveur des ouvriers et des ouvrières syndiqués(es) par rapport à ceux et celles qui ne le son pas ${ }^{(0)}$. Nous ne prétendons pas que les disparites dans les conditions de travail sont toutes attribuables a la variable "syndicalisation". Des caractéristiques reliees a la main-d'oeuvre, à l'industrie elle mềme et à la taille des etablissements ${ }^{140}$ contribuent à expliquer également ces différences.

\subsection{Le Code du travail}

Le régime actuel de reconnaissance syndi cale pose comme une des conditions d'obtention de l'accreditation l'adhésion indivi. duelie de la majorité absolue imoitié plus unl, de tous les salariésles) d'un employeur viséslesl par la requête. Une fois l'accrédi. tation obtenue, l'employeur est tenue de négocier une convention collective. Le Code du travail ne permet toutefois pas l'octroi đ'une même accréditation à un syndicat qui represententerait les salariés(es) de plus d'un employeur parce que ceci aurait pour effet d'imposer à des employeurs différents, y compris ceux d'un mème secteur d'activi tés, une negociation conjointe d'une même convention collective avec le même syndi. cat. Ceci signifie donc que pour reussir à syndiquer les emploves d'hôtels ou de res. taurants, un syndicat doit réussir à obtenir la majorité hótel par hötel, restaurant par restaurant.

II n'est donc pas surprenant que le taux de syndicalisation dans le secteur de l'hébergement et de la restauration soit, selon les statistiques les plus récentes, de $7.2 \%$ de toute la main-d'oeuvre en emploi dans le secteur ${ }^{411}$. Alors qu'on retrouve un taux de syndicalisation de $44.6 \%$ pour l'ensemble des industries manufacturieres au Québec ${ }^{(42)}$ et des taux encore bien supé 
rieurs dans les services publics. Selon des donnés que nous avons obtenues par le biais du ministère du Travail, on retrouve. rait actuellement 9,923 personnes syndiquèes dans le secteur de l'hébergement ouvert à l'année longue et 7,403 personnes syndiquées dans le secteur de la restaura- tion, y compris les tavernes. Onconstate que les hôtels de taille importante sont pratique. ment tous syndiques mais "en ce qui a trait aux etablissements de 75 chambres et moins, il he $s^{\prime} y$ retrouve pratiquement aucune activite syndicale" ia3i. De plus, le syndicalisme a très peu pénétré le domaine de la restauration. Pourquoi? Cela est dü au trés grand eparpiliement géographique des salariéslesl dans une multitude de petits eta blissements. Ainsi en 1981, on comptait au Québec 3,434 établissements hôteliers et 9,888 restaurants avec ou sans permis de vente d'alcool parmi lesquels nous n'in

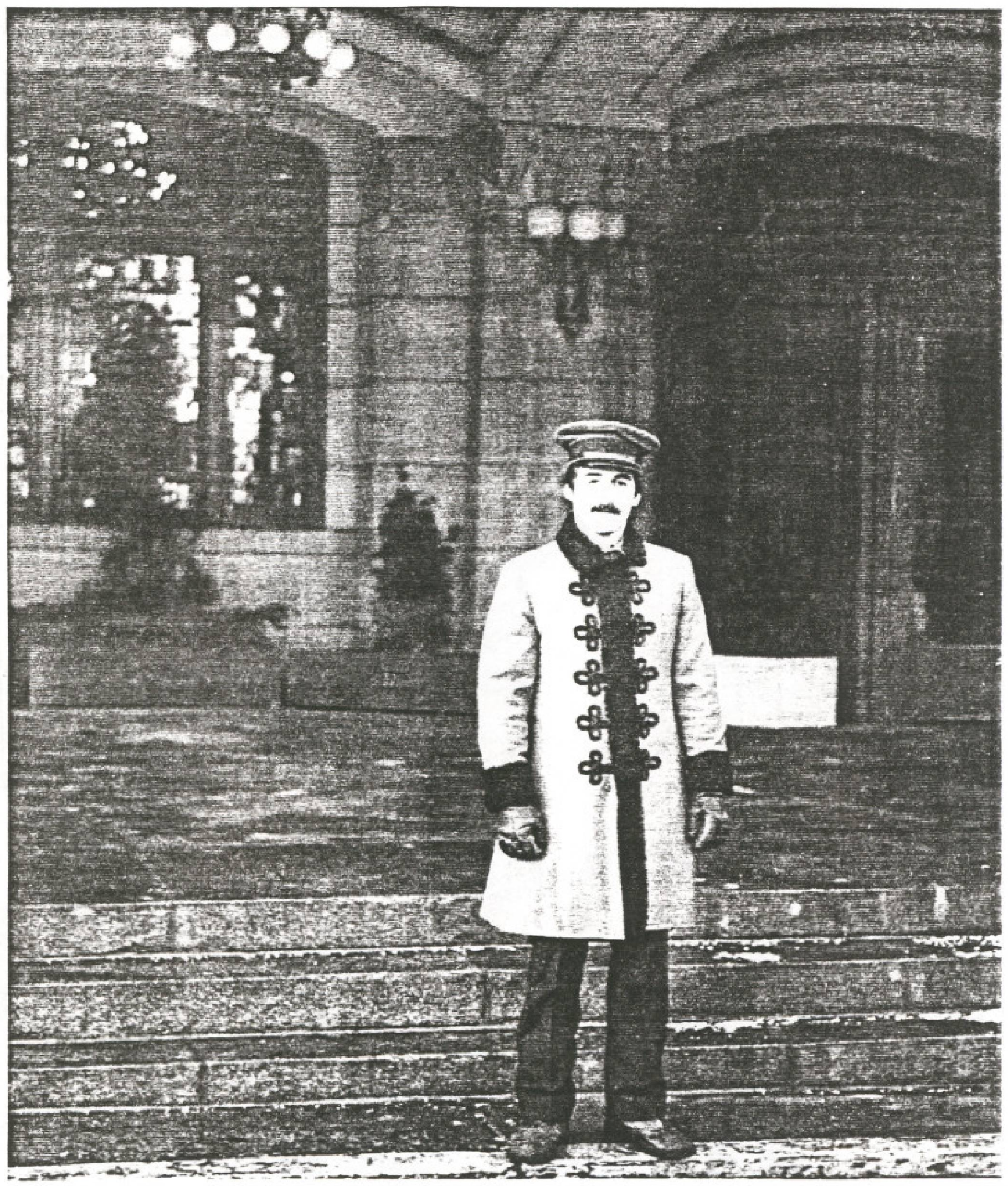


cluons pas les 2,636 tavernes, bars et clubs de nuit ${ }^{\text {i4i }}$

Ces conditions posent à tout mouvement de syndicalisation des difficultés insurmontables. Les salariésles), particulièrement ceux et celles de la restauration, travaillent en très petits groupes, de plus, ils (ellesi sont très souvent en contact direct avec le proprietaire de l'établissement, devenant ainsi très vulnérables aux possibilités d'intimidation directe ou indirecte. Les autres facteurs défavorisant l'accès au syndicalisme dans ce secteur sont reliés aux perspectives plutôt sombres quant aux chances de négocier d'une taçon efficace dans le cadre actuel du Code du travail. Les trois serveuses du restaurant "Le Roi de la patate frite" à TroisRivières qui réussiraient malgré tout à obtenir une accréditation ne seraient pas au bout de leurs peines. Leurs chances d'obtenir une convention collective satisfaisante sont plutôt minces puisqu'elles disposent d'un pouvoir de négociation trés faible et que. compte tenu de la concurrence des autres restaurants dont le personnel n'est pas syndiqué, l'emploveur lui-mème peut se trouver dans une position très precaire pout améliorer les conditions de travail de son propre personnel.

II apparait donc que la solution permettant le relèvement des conditions de travail du personnel de la restauration et des établis sements hôteliers de taille petite ou moyenne ne se trouve pas dans les dispositions du Code du travail telles qu'actuellement formulées.

\subsection{La voie des décrets et la} syndicalisation multipatronale La loi sur les décrets de convention collective constitue dejà un champ d'experience pour la négociation multipatronale. Les pourparlers préalables entre les syndicats et le regroupement patronal sur le contenu de la convention collective à extensionner consiste dejả en une certaine forme de négociation. On ne peut évidemment assimiler celle-ci à celle qu'on retrouve dans le cadre du Code du travail. En effet, le syndicat ne "represente" pas les non-syndiques et il ne dispose pas de movens pour les consulter et les regrouper; il n'a pas droit de greve non plus.

Le but d'un décret est d'uniformiser les conditions de travail de facon a ce que les employeurs ne se livrent pas à une concur. rence déloyable en baissant indüment leurs coûts de main-d'oeuvre. On retrouve d'ailleurs le régime des decrets dans des indus. tries caractérisés par l'existence d'un très grand nombre de petits employeurs lex: garages, salons de coiffure).

Plusieurs observateurs analysent la négocia tion multipatronale en puisant des comparaisons avec le système des décrets. II existe effectivement des points de similitude importants puisque le decret, comme une convention collective multipatronale pourrait le faire éventuellement, s' applique à plusieurs employeurs à l'interieur d'un secteur donnè.

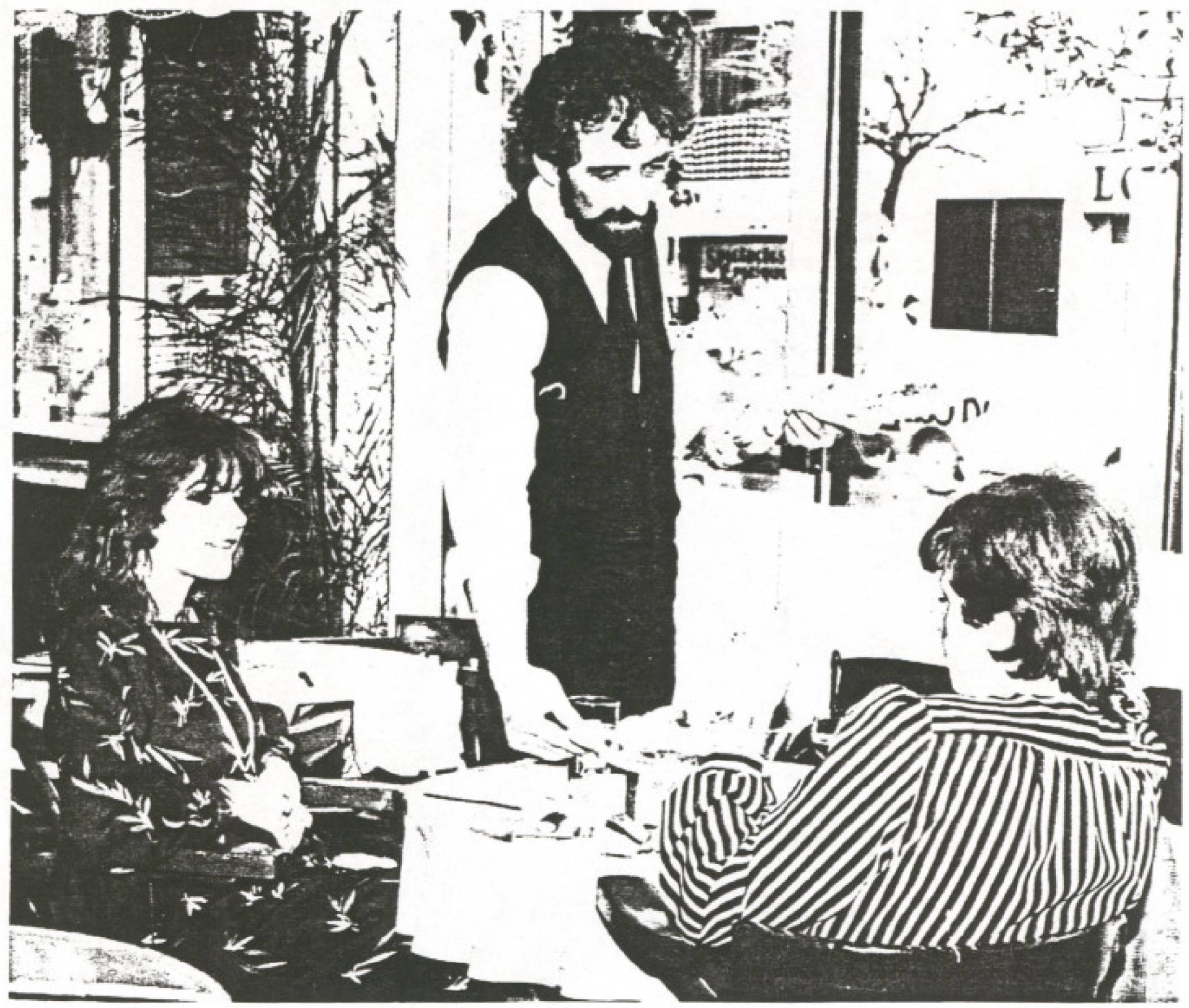


L'acréditation multipatronale est une forme de reconnaissance par une instance jurid quement competente d'une ou plusieurs association(s) de salariés(es) à l'emploi de deux employeurs difterents ou plus. Ains on pourrait retrouver à l'intérieur de la mème accréditation tous les employéslesl des res taurants de Trois-Rivières par exemple en autant que ces salariesiesi ont majoritaire ment signifié leur volonté d'étre représentés(es) par un syndicat. Cela signifie que, une fois l'accréditation obtenue. les employeurs devraient se regrouper ensem ble pour négocier une convention collective unique pour l'ensemble des salariés(es). Un tel encadrement contribue à briser le senti ment d'isolement des personnes qui travail. lent dans de petits etablissements, uniformise les coûts de main-d'oeuvre et permet un meilleur équilibre entre les forces en pré sence. Dans une telle perspective il $n^{\prime} y$ a pas d'intervention de l'Etat dans la détermination d'une éventuelle convention collec tive multipatronale; ce sont les parties elles. mèmes qui fixent le contenu de cette dernière.

II existe donc des diffetrences entre un régime de négociation multipatronale et un régime des décrets. Le gouvernement pourrait eventuellement décider d'instituer un decret dans le secteur de l'hótelierie ou de la restauration. L'expérience nous indique que le contenu d'un decret, tout en étant d'une façon gènérale superieur aux condi- tions minimales prévues ả la Loi sur les nor mes du travail, n'est pas aussi complet qu'une convention collective; ainsi la loi sur les décrets ne prévoit pas l'extension des clauses contractuéles (ex: ancienneté), puisque celles-ci "ne donnent pas ouver. ture à la concurrence ${ }^{* t} 145$ !

Malgré tout, la loi sur les décrets peut ouvtir des avenues interessantes quant à la facilitaton de l'accès au syndicalisme. Ainsi, la loi sur les décrets pourrait ètre amendée de façon à ce qu'à partir du moment oủ $35 \%$ des travailleursieuses) de la restauration ou de l'hôtellerie dans une région donnée sont membres d'un syndicat, l'ensemble des salariés(es), syndiqués(es) et non" syndiqués(es), seraient consultés(es) par scrutin secret sur leur volonté d'être repré. sentesles) par un syndicat pour des fins de determination des conditions de travail. Si la réponse est majoritairement positive, it s'agirait d'une extention de toute la convention collective, y compris les dispositions de ladite convention qui concernent l'apparte. nance au syndicat (régime syndical). L'accré ditation et la negociation multipatronale pourraient donc alors se dérouler dans le cadre d'une loi sur les decrets de convention collective.

\section{Conclusion}

Toute la demarche que nous avons suivie dans le présent texte visait à tracer un portrait sommaire des conditions de travail et de la repartition des employesles dans le secteur de l'hótellerie et de la restauration Nous avons tenté de degager une voie d'avenir quant au choix d' un mode eventue pour en arriver à hausser le niveau des con ditions de travail dans ce secteur. Nous avons choisi particulierement le secteur de I'hótelierie et de la restauration à titre d'il. lustration a cause de son importance nume rique. Cependant, la perspective que nous avons tenté de dégager pourrait s'appliquer autant pour le secteur des agences de vova ges que pour le secteur des organismes de loisirs.

Dans son economie actuelle, le Code du tra vail ne peut pas favoriser la syndicalisation, et ce contrairement aur principes èmis à l'article 3 qui reconnait le droit d'association. Le projet d accreditation et de négociation multipatronale vise á rétablir un rapport de force réel entre les parties. Nos trois serveuses de Trois-Rivières sont dans une situation impossible et mème leur employeur peut egalement être dans une position pré caire face à ses concurrents. La situation de la concurrence peut en effet rendre irrealisable l'octroi de certaines ameliorations au niveau des conditions de travail revendi. quées par le syndicat. De telles perspectives contribuent donc à decourager les tra vailleurs(euses) à se regrouper et ne peuvent qu'accroitre l'hostilite patronale a la syndi. calisation dans le cadre législatif actuel de mëme qu'inciter au recours à tous les movens pour éviter l'implantation syndicale.

\section{Références}

(1) SECRETARMAT DES CONFERENCES ECONOMIOUES.LE tounisme: perspectives de relance. Outbec, Mrusgere du Consed extestil. 1978. Pि 45

(2) OHice de la protection du consommates, Centedemigr

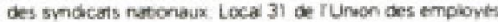
d houtels, iestautants et commis de bar. Centrace de len

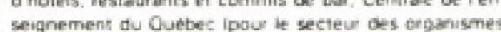

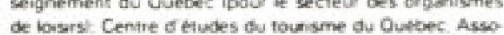
de hosss. Centre de efudes du tounsme du Outbect. Asso: des associstions toungtiques, instivt do tounsme et of he

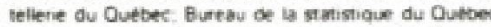

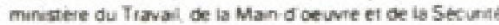

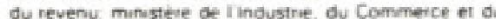
Tounume

(13) GOSSELAS K EI GAGNE, I, Situation de lindurtre hou

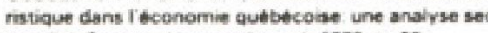
tonelis. Outbec. Unuersie Leval, 1976 o 50

(4) BaUMANA Ande et ASSOCIE5. L'induntre touristique su Qubbe: Effectits 1970 1980. besoins quantraters

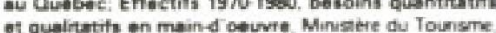

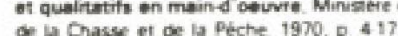

15) Von trous articles de VEILLfUX Manon ans Tourime * numpor des 16 sept 1962.30 sepr 1982 et 14 acto bre 1900

(6) OHce de lis protection du consommstes ef Pensome Gude to Canadas Travei indursily. Spong Autumn 'Ba

(7) GOSSELIN $\mathrm{K}$ e GACNE. 1, op cit, p. 27

(18) 1d. A. 24

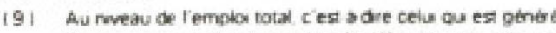

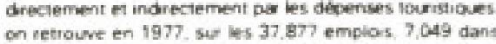

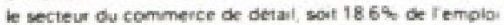

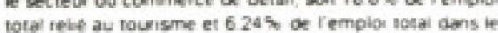

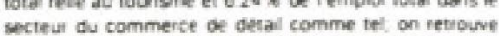

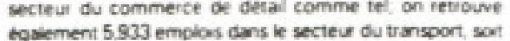
bosiement 5.933 emplos dons we secteu du transport som 15.64 de femplos volal iebe au tounsme ef 5.325 , of fem

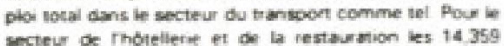

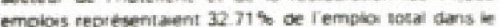
sectey comme tel
II faut bouner que hes auteus cossELin al GAGNE on bayt hur calcul sur les informations foumes par Srabst gur Consds Selon kul rasonnement, an ariverat a 43 gai

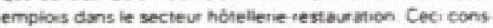

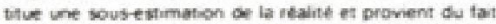

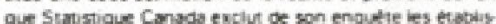
vets Q plusturs miliess de restavizits ons mons de 20 personnes a leus emplo:

(00) STATISTIQUE CANADA Emploi, gains wh durte du tra wail, Caralogue 72002 mensuel, Juiliet 1982 p. 26

611 Id. D 120 pow une exphcation os concept at metho

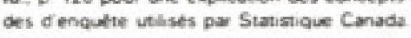

(12) Miv5TRE DU AEVENU. Livre wert aur th ahtuation su Quabec des travailieurs ot des travaillevers ay pole boirs Outher Gounernement du Ouetec 1982 o. 7

113. CENTAE DE AECMERCME ET DE STATISTIOUES SUA. LE MARCHE DU TRAVAIL. Probitemes ef perapectives d emploi dans le secteur noteiner du Cutbec, Qutdec. Geuwernement du Guebec. 1980 p. 30

(17. BUFEAU DE LA STATI5TIOUE DU GUEBEC, Stmiat

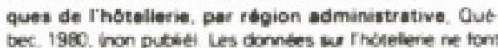

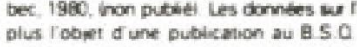

i15. MINISTAE DU REVENU op eit, 7 .

11E) CONSEIL DU STATUT DE LA FEMME Srndicalisation

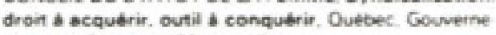
ment du Quebec. 1991. pi 171

(1) MINISTAE DU REVENU op cit, p. 7 . Nove incluons $\mathrm{E}$

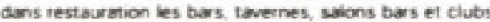

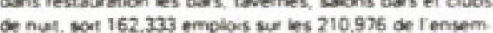

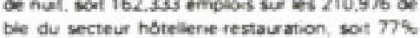

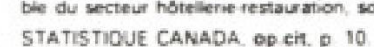

त9) CONSEIL DU STATUT DE LA FEMNE, op.cit p 171

DOI CENTAE DE RECHERCHE ET DE STATISTIOUES SUÁ LE MARCHE DU TRAYAIL OO cit. D. B.

Qi1 id. 0 6?

(2) lid 9 .

Q3! औd D का

Q4i CONSEIL DU STATUT OE LA FEMME, Op eit, P. IBE

CS. CENTAE DE RECHEACHE ET DE STATISTIOUES SUR LF WAACHE DU TAMVAAL OP EI Af
(26) It. $\bar{p} 100$

(2) id o iot

(28) id. 0 . 35

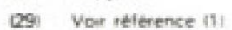

(20) STATISTIOUE CANADA op eit o 2327

BII CENTRE DE RECHEACHE ET DI \$TATISTOULS SUA LI MARCME DU TRAVAIL, OP. CIT. P 130

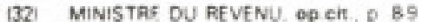

133 id. $p$. 7

(34) MINISTRE DU REVENU op ein. OS

135. CENTAE DE RECHEACHE ET DE STATISTICUES SUA LF MARCME DU TRAVAIL OP EIT. D IOJ

(b) la is 9

(97) Id. 00219220

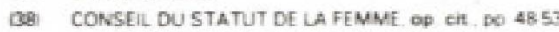
ell of 7375

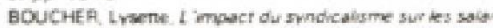
res dans te sectrur manulachuner as Quegec dans Le Marche du Traval Gueter. Cenife ge recherche er de slatistiques sur le mache du travail teriet 19g2. wol 3 no. 2 . DD 4854

da CONSEL DU STATUT OE LA FEMNE, op cin D 45

40) MARION G. LACRDX $A$ it LATRAVERSE Tante des

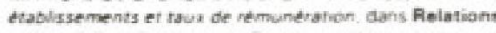

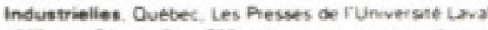
1979. woil 34 no 3. D 572 il faum remarquel que teludie os madame Lrsene Boucher chtic a is reference (3b) teet

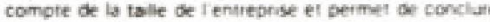

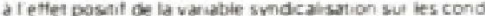
wons de salsin:

441) CONSELL DU STATUT DE LA FEMME. OP EI D 177

4421 DELORME Francoss Oueigues donnter tur it sundichal

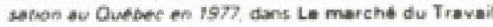

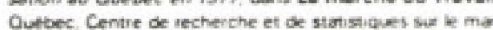
che du varal No 196. wol 1, no 1. D 35

43. CENTAE DE RECMERCHE ET DE STATISTIOUES SUR LF MAACHE DU TRAYAL OPEA D 157

(4A) MIR 5 TRE DU RFVFNU ag cit, g. 10

445) BeAULIEU. Mare Lovis. Les continta de droit dans les repports collectito du twawil Outbec, Les Perses a TUnewerste Laval 1955, ai 147 